Identidad entre ser y verdad

\title{
Las nominaciones de Dios en el argumento del Proslogion
}

God's nominations in the Proslogion argument: Identity between being and truth José Luis Gaona Carrillo

\section{(2) OpenEdition}

\section{Journals}

\section{Edición electrónica}

URL: https://journals.openedition.org/medievalista/5149

ISSN: 1646-740X

\section{Editor}

Instituto de Estudos Medievais - FCSH-UNL

Referencia electrónica

José Luis Gaona Carrillo, «Las nominaciones de Dios en el argumento del Proslogion», Medievalista [En línea], 31 | 2022, Publicado el 01 enero 2022, consultado el 02 febrero 2022. URL: http:// journals.openedition.org/medievalista/5149

Este documento fue generado automáticamente el 2 febrero 2022

\section{(c) (†) \&}

Mediavalista está licenciado com uma Licença Creative Commons - Atribuição-NãoComercial 4.0 Internacional. 


\section{Identidad entre ser y verdad}

\section{Las nominaciones de Dios en el argumento del Proslogion}

God's nominations in the Proslogion argument: Identity between being and truth José Luis Gaona Carrillo

\section{NOTA DEL EDITOR}

Data recepção do artigo / Received for publication: 31 de Março de 2021. Data aceitação do artigo / Accepted in revised form: 12 de Novembro de 2021

Introducción

El argumento del Proslogion fue un manuscrito medieval redactado por el filósofo San Anselmo de Canterbury. A lo largo de los capítulos de este texto se vertió un agudo problema relativo al ser y existencia de Dios. En este artículo serán explicados los diversos nombres ${ }^{1}$ de Dios, los cuales ponen de relieve algunos de los atributos del ser divino. Consecuentemente, en esta exposición se demostrará una reinterpretación aplicada a la lectura del Proslogion orientada por el estudio de Ricardo Oscar Díez en ¿Si hay Dios, quién es?². Deudores de su investigación, propondremos en este artículo una original postura no vista en el texto de Ricardo O. Díez, esto es, la identidad del ser del Proslogion con la noción de verdad del De Veritate, propuesta novedosa que podría vincular argumentativamente ambas obras del Arzobispo de Canterbury, remarcando la sistematicidad de su pensamiento y que el estudio de Díez no trabajó. Entonces en nuestro ejercicio hermenéutico suponemos ya una nueva lectura respecto a estas nominaciones divinas explicadas por Ricardo O. Díez. Este estudio divide por etapas el argumento anselmiano, de ahí que nos remitiremos exclusivamente a una revaloración hecha a las nominaciones o nombres que señalan esas cualidades de Dios. Sin embargo, también es importante tener presente la metodología o dialéctica anselmiana, a través de la cual San Anselmo oriento su pensamiento bajo la sentencia: fides quaerens intellectum $^{3}$. Esta sentencia puede traducirse en la fe que busca la intelección. El abad de 
Bec abrió un modo de pensamiento el cual antepone un límite a la razón de aquello que se cree por fe, en otras palabras, existe una línea epistemológica que se vuelve infranqueable respecto a la creencia que inicia el pensamiento. De este modo, las nominaciones divinas revelan racionalmente los atributos de Dios, pero sin incurrir en una excesiva racionalización de esa fe que siente el corazón. En consecuencia, el desarrollo de este artículo explicará tres momentos, donde cada uno consiste en un nombre de Dios.

3 Por otro lado, se advertirá en el transcurso de la argumentación el rasgo de verdad que posee intrínsecamente cada nominación, marcando así una identidad ${ }^{4}$ entre ser y verdad. De ahí que además del argumento del Proslogion sea conveniente tomar en cuenta otro texto vital en el pensamiento del abad de Bec, nos referimos al texto De Veritate $^{5}$. En este texto de la verdad, San Anselmo dedica algunos capítulos donde desarrolla los tipos de verdad y el cómo se va alcanzando una única definición de la suma verdad. Entonces, a nuestra interpretación, esta idea motiva a una identidad que marque el entrecruzamiento conceptual de una obra a otra, entre verdad y ser. De lo dicho con anterioridad puede ser señalada una continuidad argumentativa del texto del Proslogion al De Veritate. Finalmente, en los apartados que dividen este artículo se describirán algunos elementos característicos de los atributos de Dios propios al modo de pensamiento medieval.

Conviene señalar que las nominaciones o los nombres de Dios son pues conceptos en los que se encuentran vertidos ciertas adjetivaciones que el intelecto ha logrado con el ejercicio intelectivo ${ }^{6}$. Así en el argumento del Proslogion es importante acotar que lingüísticamente hablando, el nominar señala con las palabras el significado ${ }^{7}$ que éstas tienen con los objetos que nominan, es decir, el pensamiento mira desde el signo al objeto que alude y es a partir de ese momento que una palabra o un enunciado puede ser juzgado verdadero ${ }^{8} \mathrm{o}$ falso. Esta consecuencia, suscita la indagación de la verdad del ser finito, pero que por contraste Anselmo intenta dilucidar la definición de la suma verdad. Una de las premisas centrales a este manuscrito medieval del Proslogion ha sido remarcar la imposibilidad del no-ser de Dios quod non possit cogitari non esse ${ }^{9}$. Los estudios modernos aplicados a la Edad Media, particularmente del célebre y gran medievalista francés E. Gilson en su texto La filosofía en la Edad Media ${ }^{10}$ nos ha hecho ver una interpretación ontológica, más que un pequeño opúsculo dirigido a los monjes que Anselmo tenía a su encargo. De este modo, este argumento a lo largo de la historia de la filosofía ha sido clasificado en tanto argumento ontológico, y específicamente de un tipo de ontología "realista". Conviene advertir que dicha interpretación es un modo distinto por el cual el Arzobispo de Canterbury nunca se pronunció al respecto.

Los textos medievales no terminaron por acoger las categorizaciones modernas atribuibles a las etiquetas realistas/nominalistas que los estudios modernos les suelen atribuir. Por ende, una consecuencia natural de estas interpretaciones modernas, ha sido traer a la discusión la oposición frecuente entre la conocida dialéctica y antidialéctica; posturas fuertemente remarcadas por el propio É. Gilson y que a lo largo de la historia de la filosofía del Siglo XX ha dejado su huella bajo las etiquetas "realistas o nominalistas" ${ }^{11}$. Estas vertientes antagónicas están íntimamente ligadas a la cuestión del lenguaje, en este caso, a las nominaciones de Dios, sin embargo, también es preciso no perder de vista la impropiedad de querer subsumirlas estrictamente a una cuestión lingüística, ya que como veremos también existe una repercusión ontológica y epistemológica en el plano de la realidad. 


\section{Primera nominación de Dios - Te esse aliquid quo nihil maius cogitari potest ${ }^{12}$}

5 El primer nombre de Dios Te esse aliquid quo nihil maius cogitari potest ${ }^{13}$ aparece en el capítulo II del Proslogion, apartado que inaugura el inicio nominativo de este itinerario filosófico/teológico. Como podrá observarse en esta sentencia, el ser de Dios está referido al pensamiento en términos negativos, es decir, aquello mayor que lo cual nada pueda pensarse antepone un máximo al pensar. De ese nihil que niega al pensamiento un ser mayor a él, es sumado el adjetivo maius, adjetivo calificativo que constata la unidad mayor de un solo ser. Por su parte la univocidad ${ }^{14}$ es una propiedad exclusiva de un solo ser, id est, no puede ser propiedad de algún otro ser tal que lo rebasase, pues ya no se trataría del mayor que lo cual nada pueda pensarse. Cualquier otro objeto (res) no puede estar por encima de éste, de ser esto falso, podría encontrarse un ser tal que rebasase al ser de Dios, lo cual, es contradictorio. Nótese pues, el primer atributo de Dios en este capítulo II del Proslogion. Hemos de reflexionar entonces en el valor de verdad $^{15}$ que encierra este primer momento del maius divino. Efectivamente, es concedido que no puede existir un ser mayor a Dios, entonces este ser debe ser un máximo a todo ser en comparación de cualquier otra creatura. Si ha de ser el mayor, debe ser verdadero, puesto que es mayor (maius) ser verdadero que falso. Este rasgo veritativo en la identidad aquí sugerida remite al otro texto anselmiano aludido: De Veritate $^{16}$. La identidad o relación recíproca entre el ser de Dios y la verdad de él se desenvuelven co-sustancialmente.

El capítulo II del De Veritate habla precisamente del carácter veritativo que llega a tener la significación de las palabras. Si seguimos con atención el diálogo expuesto en este texto filosófico encontraremos un tipo de verdad que se asemeja al rasgo ontológico de ese ser mayor del cual nada pueda pensarse, esto en el sentido de la rectitud de las palabras. Habrá que recordar la réplica que esgrime el abad de Bec a Gaunilón justo en el momento a través del cual demuestra que los pensamientos falsos e inexistentes no pueden ser entendidos por el entendimiento, entonces, los pensamientos verdaderos si puede ser entendidos por el entendimiento: "M.-Quid igitur tibi videtur ibi veritas? D.Nihil aliud scio nisi quia cum significat esse quod est, tunc est in ea veritas et est vera." ${ }^{17}$

7 Ahora bien, en este capítulo II del De Veritate, a nuestro juicio, es dada una continuidad discursiva con el capítulo II del Proslogion, razón que sugiere hablar de esta identidad entre ser y verdad en Dios. Una lectura atenta de ambas obras, permite mostrar esta semejanza marcada que se va dando de un texto a otro, pese a que San Anselmo redactó las obras con varios años ${ }^{18}$ de diferencia. En efecto, dichos manuscritos tratan temáticas diferentes, sin embargo, los elementos relativos al ser y verdad de Dios siguen estando presentes en ambos y más aún coinciden en las referencias que hemos citado. En otras palabras, desde el ejercicio del pensamiento se hace efectiva la constatación acerca de la imposibilidad del no-ser divino. Esta no-posibilidad queda calificada con el maius que es verdadero. Siguiendo el mismo orden expositivo del Proslogion, así de este estudio aplicado por Ricardo O. Díez, el capítulo III explica el momento de realidad de esa palabra unificada con el ser:

"el capítulo III refiere a la realidad o esse divino que la palabra señala. Se actúa una

descripción a partir de las cosas mismas [...] es el esse divino que intenta responder 
a la primera cuestión de la síntesis que el autor hace en el prólogo (prooemium) Quia

deus vere est"19.

Esta nominación no solo es el inicio de las restantes, está presuponiendo el contenido de fe por el cual Anselmo constataba desde la plegaria alcanzada al inicio del texto del Proslogion. La intelección de la fe inicia su camino hacia un proceso dialéctico sintetizando palabra-ser, resultando conveniente no perder de vista la sentencia anselmiana por excelencia: fides quaerens intellectum. Sea conveniente decir que Anselmo confiaba tanto en las Sagradas Escrituras ${ }^{20}$ que por ello no temía en llamar a su esclarecimiento racional, siempre sin agotar eso que su corazón cree. A este punto se abre una gran discusión que varios estudios especializados en la filosofía anselmiana no han convenido en acordar. Más allá de entrar en el debate, sencillamente sea de paso resumir la postura del Arzobispo de Canterbury. San Anselmo como creyente nunca puso en duda la existencia y el ser de Dios. Las posibilidades dadas por la racionalización o intelección de la fe vienen dadas por el ejercicio meditativo-reflexivo posterior a la confesión de fe. Entonces este trayecto racional especulativo de la primera nominación del Proslogion recorre del capítulo II al capítulo IV una frontal respuesta a la figura que encarna al insensato, es decir, quién niega a Dios. Por lo tanto, esta búsqueda racional entra en discusión directa hacia la réplica que formula aquel que habla en nombre del insensato ${ }^{21}$. Recuperando el sentido de la cita textual anterior (respecto al capítulo III), nótese el componente veritativo que se desenvuelve en conjunto con el verbo intransitivo de ser: quia deus vere est. Si Dios verdaderamente es, entonces enlaza la conjunción verdad-ser; ella misma admite mutua reversibilidad del ser a la verdad, es decir, ser-verdad. Por lo tanto, puede señalarse una paridad discursiva e identitaria entre el ser alcanzado por el Proslogion y la definición ${ }^{22}$ de verdad vertida en De Veritate.

9 Este es un buen ejemplo de la capacidad visionaria que tiene la filosofía anselmiana al poner de relieve la mutua interacción gestada entre el entendimiento y su relación valorativa de la verdad al ser de Dios. Lo decisivo, es hacer notar que no se trata exclusivamente de una verdad tal que solo remita a Dios. Los elementos aquí ofrecidos miran más ampliamente la verdad intrínseca a la realidad misma que ha sido creación divina. Este orden creador no deja posibilidad alguna de ruptura o lugar a la falsedad del objeto (res). En vista de lo anterior, cuestionar el valor de verdad que tienen los objetos han hecho lícita una lectura más general del argumento del Proslogion orientándolo en su discurrir hacia una perspectiva ontológica. Entonces, no considerar todo el entramado discursivo que tiene esta primera nominación con las subsiguientes nominaciones hace del argumento un pequeño fragmento racional acerca de la existencia de Dios, lo cual, desde la perspectiva aquí señalada incide en una interpretación parcial y sesgada a la unidad del mismo argumento.

10 A consecuencia de esto muchos estudios interpretativos comúnmente tienden a limitar sus observaciones a esta primera nominación, fracturando la totalidad que representa todo el argumento en sus 26 capítulos. Particularmente dicha división queda motivada por considerar solamente los capítulos del II al IV, capítulos donde la mayoría de estos estudios convergen en sostener el denominado "paso" del plano lógico al ontológico ${ }^{23}$; movimiento que de alguna manera inauguró Kant en su intento por refutar al argumento anselmiano en su contenido en su Crítica de la razón pura ${ }^{24}$. Sin embargo, pocos análisis filosóficos con diferentes perspectivas han puesto en diálogo esta 
contrariedad demostrando que reducir el argumento a los capítulos aparentemente centrales agotan la intención primordial de San Anselmo al redactar este opúsculo.

11 Respecto a los estudios interpretativos de este manuscrito medieval Julián Velarde Lombraña en su investigación introductoria a una edición del Proslogion ${ }^{25}$ acierta en resaltar dos cuestiones presentes de modo genérico a las diversas indagaciones aplicadas a este texto medieval:

"El análisis del argumento exige ante todo, aclarar las dos cuestiones siguientes: (1) ¿Qué dice probar el argumento? (2) ¿Prueba el argumento lo que dice probar? La primera cuestión es qué significa el vere esse que aparece en varias formulaciones, a nuestro entender, relacionadas, como: quia Deus vere est (Proemio); quod vere sit Deus (cap. II); vere es; habes esse verissime, habet esse non sic vere (cap. III). Las interpretaciones (y, por consiguiente, las traducciones) divergen en función de la interpretación general de la obra anselmiana. Así, por ejemplo, en la interpretación fideísta de Barth, el fundamento de la discusión es la fe, y «una vez presupuesto que es verdad que Dios existe, que Dios es el ser supremo, que él es un solo ser en tres personas, que él se ha hecho hombre, etc., Anselmo discute la cuestión de saber cómo eso es verdad»" 26 .

12 A pesar de estas palabras J. V. Lombraña añade: "Por lo tanto, no se trata de una prueba de Dios, sino de reconocer que Dios existe verdaderamente (vere est). (...) La noción de vere es, efectivamente fundamental en el argumento del Proslogion, así como en las objeciones al mismo" ${ }^{27}$. Este elemento veritativo anunciado en el proemio permite pensar más que en una demostración de Dios, es una respuesta donada de Dios al hombre, particularmente un gozo ${ }^{28}$ que recibió Anselmo en su respuesta a la plegaria que ofreció. Dicho de otra manera, la verdad del ser de Dios es el punto de inicio donde queda sostenido todo el argumento. Esta primera enunciación parte de un ser tal que es verdadero, que es mayor (maius) y por el cual no puede haber otro ser que sea por encima a éste.

Ahora bien, la cuestión relativa a la postura que sostiene el insensato consiste en negar la primera nominación expuesta por Anselmo, sin embargo, comete el equívoco de aplicarlo al mayor ${ }^{29}$ a todas las cosas. Brevemente recordemos que en la Edad Moderna temprana así de la Edad Moderna tardía, los filósofos racionalistas en su recepción al argumento anselmiano, y más especialmente René Descartes inscribiría tanto en Discurso del Método, como de sus Meditaciones Metafísicas su "demostración de la existencia de Dios". Pese a que no haga ninguna referencia explícita al Abad de Bec, Ricardo O. Díez sostiene esta modalidad moderna del argumento de San Anselmo. De modo que el filósofo francés hace de este rasgo de perfección una co-sustancialidad con el ser de Dios, pero que como ya hemos denunciado previamente, equiparó este atributo divino al total de la capacidad intelectiva humana. En otras palabras, el que niega aquella mayor que lo cual nada pueda pensarse, no solo niega al ser perfectísimo a toda creatura, confunde el recto significado de estas palabras sobreponiéndolo en un mismo "nivel ontológico" con el resto de las creaturas asumiendo que es el mayor a todas las cosas, lo cual como puede percatarse es distinto. En sentido estricto, ésta es la vía que edifica la réplica de Gaunilón a San Anselmo. Se trataría de un ser que no señala a Dios, más bien, imputa el maius a un ser distinto del sumo ser, configurándolo en un sentido distinto al de esta primera nominación. A fin de cuentas la réplica de este monje Gaunilón no puede sostenerse ¿Cómo podría nominarse algo que superase aquello mayor que lo cual nada pueda pensarse? Suponiendo que ha sido "posible" pensarse o inclusive negarse aquello mayor que lo cual nada pueda pensarse, la misma réplica de Gaunilón extrae desde la misma negación a la sentencia anselmiana su 
formulación propia; pero justamente ya no se trataría de aquello mayor que lo cual nada pueda pensarse, lo cual es absurdo.

Resumiendo, aquello mayor que lo cual nada pueda pensarse no puede ser una invención o creación conceptual del pensamiento humano. El maius antepone una distinción ontológica respecto al resto de las creaturas que no pueden situarse por encima a este ser perfecto que es Dios. A la par, en el proemio a este argumento es desarrollada una premisa que sella esta nominación con el valor de verdad. Al modo de pensar de este artículo, se da una identidad con el ser, en medida que puede tomar una mutua reversibilidad entre sí misma; una verdad que es y un ser que es verdadero. Este momento despliega el preludio a un llamado de la verdad en aquello que se cree. Es el inicio a un recorrido más extenso que mirará el pensamiento humano en su esfuerzo intelectivo por explicar aquello que cree por fe. El maius de aquello mayor que lo cual nada pueda pensarse contiene sustancialmente una cualidad negativa. Esta negatividad del pensamiento permite asumir que ninguna otra creatura puede imponerse al ser mayor que lo cual nada pueda pensarse. Lo mayor se impone en esta primera nominación al intelecto y a la realidad.

Por otra parte, la contemplación de las cosas (res) corrige los abusos o sinsentidos dados por el ejercicio exclusivo del pensamiento, es decir, el pensamiento necesita constatar lo inteligido con la experiencia directa de lo real. Este contacto directo con la realidad ${ }^{30}$ comprueba al intelecto los modos de ser distintos de las cosas (res), y a su vez permite unificar o romper la posible unión de lo pensado con lo experimentado; concluyendo si hay o no correspondencia. De suerte que el pensamiento al interpretar la palabra conjuntamente con la visión de la cosa, permite juzgar mediante una meditación contemplativa su valor de verdad: "El pensar mira hacia las cosas para corregir su palabra y hacia las palabras para entender cuándo significan correctamente a las cosas en lo que son, esto es, para entender la realidad de lo que es, realidad que, en este caso, es el ser mismo de Dios" ${ }^{31}$.

Presentada esta primera nominación proviene la necesidad de indagar sobre la próxima etapa en la llamada segunda nominación. Este próximo paso recorrerá una ascensión aún mayor que la anterior.

Segunda nominación de Dios - summum omnium ${ }^{32}$

18 El capítulo III puede entenderse en tanto mediación hacia la segunda nominación de Dios vertida en el capítulo V. El título de este apartado tercero explica la imposibilidad ontológica del no-ser de Dios, es decir, se trata de un rasgo ontológico del ser de Dios al que no le conviene la negación de sí mismo, pero no solo ontológicamente hablando de su realidad efectiva, sino también, en la imposibilidad por la cual el pensamiento pudiese entender lo que no es: Quod non possit cogitari non esse ${ }^{33}$. Esta afirmación dilucida en el fondo la característica respecto a la eternidad de Dios, pues Dios siempre es, y a éste no puede convenirle el no-ser. Caso contrario, cualquier creatura se pondría por encima del ser de Dios, pero recordemos que todo ser finito participa de una contingencia ontológica, con principio y un fin. Ahora bien, la intención de San Anselmo en este capítulo III ha sido nuevamente dar una mayor complejidad al pensamiento intelectivo al llevarlo hasta un límite mayor. Este nuevo derrotero llega hasta una barrera infranqueable donde resulta imposible pensar el no-ser de Dios. Por lo tanto, Anselmo concluye que no puede pensarse que no sea. Particularmente, en este punto de la discusión con el insensato, es quién neciamente intenta negar este ser de Dios, pero como ya se ha explicado, no es posible tal afirmación. 

Anselmo conceptualizar una nueva adjetivación de Dios mediante el adjetivo melius. El título del capítulo V del Proslogion anticipa a la argumentación esta cualidad de "ser mejor": "Quod Deus sit quidquid melius est esse quam non esse; et solus existens per se, omnia alia factia de nihilo" ${ }^{34}$. Conforme a esta segunda etapa puede notarse cierta analogía discursiva con la primera, no obstante, este adjetivo melius impregna de una fuerza mayor al maius alcanzado previamente. Entonces, es mejor ser que no ser; en Dios no puede existir o pensarse un no-ser, más aún, es mejor ser eternamente que ser finito. La jerarquización ascendente del ser, no puede quedar restada en grado al sumo ser. Anselmo en este capítulo V nomina por segunda vez a Dios: "summum omnium"35. Por esto, el sumo ser a todas las cosas es la segunda nominación. Implícitamente tiene el melius y el maius al estar por encima de todas las cosas. A pesar de lo ya explicado con anterioridad ¿Por qué todavía el insensato no acepta en su corazón a Dios? Analicemos con detalle las palabras del Santo Doctor: “¿Por qué entonces el insensato ha dicho en su corazón: No hay Dios, cuando es tan fácil a un alma racional comprender que existe más realmente que todas las cosas? Precisamente porque es insensato y $\sin$ inteligencia" ${ }^{36}$.

Previo al capítulo V Anselmo entrevía esta decisiva respuesta:

“Existes, pues joh Señor, Dios mío!, y tan verdaderamente, que no es siquiera posible pensarte como no existente, y con razón. Porque si una inteligencia pudiese concebir algo que fuese mejor que tú, la criatura se elevaría por encima del Creador y vendría a ser su juez, lo que es absurdo. Por lo demás, todo, excepto tú, puede por el pensamiento ser supuesto no existir. A ti sólo, entre todos, pertenece la cualidad de existir verdaderamente y en el más alto grado" ${ }^{37}$.

En esta cita textual se puede advertir el cómo dos de los conceptos esenciales a argumento del Proslogion, el ser y la verdad se desenvuelven y corresponden conjuntamente con las dos adjetivaciones hasta ahora enunciadas. El maius y el melius edifican una etapa constitutiva de los atributos de Dios que el pensamiento ha inteligido, dotando de contenido al ser de Dios. Estos contenidos expresados en adjetivos permiten pensar en un "momento positivo" 38 respecto al esse divino, pues en los capítulos subsiguientes se realizará el ejercicio contradictorio a ellos ante el exceso que rebasa al pensamiento; así, estas etapas son explicadas por Ricardo O. Díez. Este especialista en la filosofía anselmiana explica ${ }^{39}$ esta importante cuestión de la segunda etapa que va de los capítulos V al XIV del argumento anselmiano. Por otra parte, la metodología o "dialéctica" del fides quaerens intellectum muestra en justa medida el cómo se van desarrollando estos logros intelectivos de aquello que se cree por fe. Lo realmente notable de esta sentencia es la tensión que se va logrando entre los propios 
atributos de estas dos nominaciones. El esclarecimiento de la fe se ha ido edificando por el pensar.

Retomando la identidad aquí propuesta entre el Proslogion-De Veritate, si bien es cierto, que el texto anselmiano sobre la verdad es ajeno a las nominaciones del Proslogion, es decir, éste no conviene en advertir algo de ellas, si puede decirse que la primera etapa del Proslogion no deja de insistir desde el proemio sobre la verdad de Dios. En esta interpretación puede construirse un enlace o vínculo entre la primera y segunda nominación por la verdad que tiene el ser divino. En efecto, era común en el pensamiento medieval no dudar acerca de la existencia de las cosas, ya que han sido creación de Dios. Él no puede crear cosas falsas o no verdaderas, ergo, lo real existe verdaderamente. Por ende, puede cuestionarse ¿Cómo podría no ser la realidad cuando ella misma está existiendo? El pensamiento humano tiene que realizar la intelección intentando unificar con la razón aquello que se tiene o se "percibe" de la cosa, juzgando posteriormente su valor de verdad. Anselmo nunca dejó de insistir en esa capacidad intelectiva que busca la verdad; fruto de una aguda meditación aplicada a la palabra. Como consecuencia se da una fase especulativa de la teoría a la realidad:

"Se ha pasado en el discurso la exterioridad de la palabra (c. II) a la de la cosa (c. III) superando dos contradicciones posibles en el pensamiento. La primera, al escuchar la palabra entre el maius contenido en el discurso del creyente y el modo de entender lo escuchado. La segunda, al adecuar lo dicho con el esse de la realidad (res). El decir de la fe y la cosa anteceden al pensamiento y lo configuran sin contradicción cuando éste entiende que lo significado por la palabra es conforme a lo que se ve en la realidad. La armonía entre esos elementos permite al pensamiento una correcta expresión" 40 .

En esta exposición interpretamos la intelección en tanto una acción racional que a partir de aquello que recibe por la impresión de los sentidos intenta unificar el contenido significativo de la palabra (concepto) con la cosa (res) percibida. Lo real no es producto de un esfuerzo intelectivo, en otras palabras, la realidad no es una creación gestada por y en el cerebro. Puede decirse que estas dos primeras nominaciones refutan o salvaguardan la coyuntura de no atribuirle mera existencia conceptual a Dios, desde el pensamiento de lo humano. Por esto es necesaria la experiencia de la cosa (res) al momento de intentar unificarla ${ }^{41}$ con el pensamiento que juzga lo visto. Suponer el modo contrario, sería asumir por el entendimiento una postulación de la razón pura que no ha probado o conocido esa realidad, agotándose en un mero ejercicio intelectivo.

De esta segunda nominación del capítulo $\mathrm{V}$ del Proslogion, puede concluirse que el ser sumo a todas las cosas, que es mayor (maius) y mejor (melius) no puede convenirle el no ser, pues si existiese la eventualidad de pensar y que además fácticamente sea dado el no ser de Dios, éste se daría en menor grado de existencia, lo cual, es contradictorio. Por consiguiente, el intelecto no puede alcanzar a inteligir su no existencia en el plano de la realidad. Anteriormente se comprobó con la figura del insensato, el cómo éste niega el ser de Dios, pero solamente su pensamiento termina contradictorio, ya que rompe la unidad entre la palabra y el orden de lo real (in re). Suprimir tanto la experiencia vital o la intelectiva restaría al adverbio de cantidad explicitado por ese maius el ser sumo a todo. La realidad se nos dona mayor a las palabras, ascendiendo de entre los capítulos anteriores, esto es, de los capítulos II-III al cap. V.

Sintetizando hasta ahora lo explicado pueden esbozarse tres momentos de estas nominaciones ${ }^{42}$. 
fronteras ${ }^{50}$ dadas entre fe y razón. Suponer un alcance absoluto del pensamiento hacia el ser de Dios, agotaría toda posibilidad del creer; ésta se tornaría irrelevante. San Anselmo consciente de su confesión de fe y además reconociendo el máximo de este pensamiento muestra que dicha frontera solo puede traspasarse mediante la experiencia religiosa ${ }^{51}$. Este tercer nombre de Dios media entre un movimiento anterior y posterior del proceso intelectivo. Del momento previo, son alcanzados los dos atributos ya señalados por las dos nominaciones, del capítulo II y del capítulo $\mathrm{V}$ del Proslogion. Así, la etapa posterior queda inaugurada por el capítulo XV hacia el excedente racional que ya no alcanza a explicar con las palabras. Es por ello que existe un llamado a la fe, invocación a causa de la improcedencia racional. El encuentro con Dios, es el encuentro con la verdad.

El misterio divino impone al pensamiento humano el rasgo de inefabilidad que la palabra del hombre se torna muda frente a lo revelado. Entonces intelectivamente comprende que Dios es la verdad ${ }^{52}$, verdad tal que puede ser escuchada, entendida y meditada. No obstante este exceso a la razón configura el aspecto de ininteligibilidad que le resulta al pensamiento imposible en su proceso intelectivo, negando a su propio lenguaje su capacidad expresiva. En los capítulos posteriores el abad de Bec explicará

Medievalista, 31 | 2022 
como Dios es esa luz inaccesible que ilumina desde sí toda parcialidad finita. Una analogía puede explicar con mejor detalle este exceso: La visión humana es incapaz de mirar directamente al sol, sin embargo, no significa que el ojo no pueda percibir de modo parcial su luminosidad, de hecho, la vista es gracias a la luz emitida por este astro solar, iluminando consigo toda la realidad. En esta analogía, ese desborde luminoso es el poder que excede al pensamiento, empero, "la visión" que se puede obtener de Dios es la experiencia ininteligible a esta búsqueda intelectiva. Esta desemejante manera es lograda por el sentir del corazón, es decir, se puede hablar de una experiencia mística o experiencia revelada.

37 A manera de conclusión, esta tercera nominación expresa ese exceso intelectivo edificando una vía distinta trazada a las dos anteriores (primera y segunda nominación). La experiencia mística o revelada no termina por explicar con el lenguaje racional ese encuentro con Dios. Pero, dicho desborde racional se explica con más detalle con las nominaciones subsiguientes, es decir, las nominaciones reveladas.

\section{Cuarta y quinta nominación}

La cuarta y quinta nominación merecen un trato aparte respecto a las tres primeras, por lo que ambas pueden explicarse en conjunto ${ }^{53}$. Tanto la cuarta como la quinta nominación son nombres divinos, debido a que se trata de la Palabra revelada por Dios. La palabra humana con la Palabra divina es distinguida por el sentir del corazón, por gracia. Se advirtió al inicio de este artículo el momento del ejercicio intelectivo que buscaba explicar racionalmente los diversos atributos de Dios. No obstante, habrá que terminar de completar el recorrido que el intelecto inauguró pero que ya no ha podido explicar. Ricardo O. Díez señala en esta cuarta y quinta nominación la donación dada por Dios.

Por lo tanto, en su interpretación mantiene la idea de considerar al argumento del Proslogion como un "librito" donde San Anselmo intentó compartir el gozo con los hermanos que tenía a su cargo en el monasterio. Este gozo que recibió una noche ${ }^{54}$ se dio cuando Dios le mostró estas nominaciones. De hecho, se trató de una respuesta al pedido que con gran fervor clamaba el Santo Doctor. Considerar entonces el argumento del Proslogion bajo este entendido, sugiere que deba realizarse una lectura completa del argumento prestando especial atención a las motivaciones del autor medieval; no reduciéndolo a una postura meramente racional y/o a unos cuantos capítulos como habitualmente se le ha reinterpretado. Si entonces este "librito" es un ejemplo de gozo y encuentro con Dios, dicha relación es dada desde el diálogo abierto desde el proemio con este ser mayor que lo cual pueda pensarse. Subsiguientemente, ve cumplida su respuesta al pedir en los capítulos XXII y XXIII. Es en estos capítulos donde precisamente se inscriben las nominaciones divinas. La búsqueda a Dios termina siendo satisfecha una vez superada la impotencia del pensamiento. Este nuevo modo revelado completa la negativa irresuelta de la tercera nominación. Si el ser que es mayor que lo que puede pensarse era incapaz de expresarse lingüísticamente, los atributos divinos en el modo revelado hacen comprensible el misterio desconocido.

Es en el capítulo XXII del Proslogion donde está expresada la cuarta nominación: “Tu solus ergo Domine, es quod es, et Tu es qui es" 55 . Este nombre recuerda las palabras del libro del Éxodo $(3,14)$ contenidas en el Antiguo Testamento ${ }^{56}$. La respuesta que Dios donó a Moisés: "Yo soy el que soy" ${ }^{57}$. San Anselmo de distinta manera cambia el primer nombre 
del singular por la segunda persona: "Tú eres el que eres"58. En el pasaje bíblico se mantenía un diálogo vivo entre creatura y creador. Análogamente puede decirse que San Anselmo inauguró el primer paso a este diálogo desde el capítulo I al pedir una respuesta a Dios. Por lo tanto, esta nominación marca el momento que ve satisfecho su pedir. La búsqueda intelectiva es superada por la verdad revelada. A este punto, la significación de las palabras coincide absolutamente con la verdad; por de ser Palabra de Dios.

41 Siguiendo el ritmo de este discurso anselmiano, la quinta nominación responde al nombre de la trinidad: padre, hijo y espíritu santo. El capítulo XXIII del Proslogion lo dicta del siguiente modo: "Quod hoc bonum sit pariter pater, et filius, et spiritus sactus; et hoc sit unum necessarium, quod est omne et totum et solum bonum" 59 . Resta decir, que Ricardo 0. Díez detalla el cómo en pueden encontrarse las palabras del Nuevo Testamento ${ }^{60} \mathrm{en}$ el Evangelio según San Juan. La promesa de Dios a los hombres es concluida con una segunda cita bíblica, inscribiendo nuevamente el diálogo creatura-creador.

“(...) el diálogo entre el hombre y Dios es posible, no por lo humano que hay en él sino por la promesa enunciada por la misma Palabra de Dios que lleva por nombre Jesús, quien nos ha manifestado: "Pedid y recibiréis para que vuestro gozo sea pleno (petite et accipietis tu gaudium vestrum sit plenum)" (Jn 16,24)"61.

El exceso es tal que el intelecto por sí solo no hubiese llegado a dicho resultado en su ejercicio meramente racional. Esta Palabra es similar a la analogía de la visión respecto al sol. La incapacidad del ojo humano en su acto perceptivo no alcanza a contemplar plenamente su totalidad. Esta Palabra de Dios es una palabra revelada real y creadora. Sin principio ni fin dicta desde el comienzo la continencia del ser inaugurando un contraste entre lo finito y la eternidad. Por esta última razón es importante el proceso intelectivo de lo humano, pero siempre reconociendo el límite de aquello que cree el corazón. Esta cuarta y quinta nominación satisfacen el pedido de la carencia humana, restituyendo todo el dolor la incertidumbre y falsedad irresuelta por la contingencia finita.

\section{BIBLIOGRAFÍA}

Fuentes primarias

DESCARTES, René - Discurso del método. Madrid: Espasa Calpe, 2003.

DESCARTES, René - Meditaciones metafísicas. Madrid: Espasa Calpe, 2003.

EADMERO - "Vida de San Anselmo". In SAN ANSELMO - Obras Completas. Vol. I. Madrid: BAC, 2008, pp. 5-73.

KANT, Immanuel - Crítica de la razón pura. Barcelona: Gredos, 2014.

SAN ANSELMO - “Acerca del gramático”. In SAN ANSELMO - Obras Completas. Vol. I. Madrid: BAC, 2008, pp. 441-443. 
SAN ANSELMO - "Carta sobre la encarnación del Verbo". In SAN ANSELMO - Obras Completas. Vol. I. Madrid: BAC, 2008, pp. 683-688.

SAN ANSELMO - “De Veritate". In SAN ANSELMO - Obras Completas. Vol. I. Madrid: BAC, 2008, pp. 487-535.

SAN ANSELMO - "Fragmentos sobre filosofía del lenguaje. Sobre el poder y la impotencia, la posibilidad y la imposibilidad, la necesidad y la libertad.” In ANSELMO DE CANTERBURY Fragmentos sobre filosofía del lenguaje. Bogotá: Ediciones Uniandes - CESO, 2001, pp. 159-211.

SAN ANSELMO - “Monologion”. In SAN ANSELMO - Obras Completas. Vol. I. Madrid: BAC, 2008, pp. 185-347.

SAN ANSELMO - Obras completas, 2 vols., texto latino de la edición crítica de F. S. Schmitt, introducción y traducción al español a cargo de J. Alameda, O. S. B., Madrid: BAC, 2008.

SAN ANSELMO - "Proslogion”. In SAN ANSELMO - Obras Completas. Vol. I. Madrid: BAC, 2008, pp. 353-441.

SAN ANSELMO - Proslogion. Con las réplicas de Gaunilón y Anselmo. Madrid: Tecnos, 2009.

SAN ANSELMO - "VI Suma teológica anselmiana sacada de las obras del santo doctor". In SAN ANSELMO - Obras Completas. Vol. I. Madrid: BAC, 2008, pp. 155-172.

Estudios

ÁLVAREZ, G. Mariano - "Sentido de la validez del argumento «ontológico» de San Anselmo". In SAN ANSELMO - Proslogion. Con las réplicas de Gaunilón y Anselmo. Madrid: Tecnos, 2009, pp. 13-31. ANTUÑANO, Alea - "San Agustín, arquitecto del pensamiento cristiano". In SAN AGUSTÍN Confesiones-Contra los académicos. Madrid: Gredos, 2012, pp. IX-LX.

CASTAÑEDA, Felipe - "Introducción a la filosofía del lenguaje en Anselmo de Canterbury". In ANSELMO DE CANTERBURY - Fragmentos sobre filosofía del lenguaje. Bogotá: Ediciones Uniandes CESO, 2001, pp. 13-152.

CORTI, Enrique - Oír, entender, argumentar. Lectura de Proslogion y De Grammatico de Anselmo de Canterbury. Buenos Aires: Miño y Dávila Editores, 2016.

GARCÍA DE LOMAS MIER, Josemaría - "El Intellectus Fidei en San Anselmo de Canterbury: claves hermenéuticas de las relaciones fe-razón en el método intelectual anselmiano". Cuadernos de Filosofía, Excerpta e dissertationibus in philosophia 4 (1994), pp. 97-185.

GILSON, Étienne - El realismo metódico. Madrid: Rialp, 1974.

GILSON, Étienne - La filosofía en la Edad Media. Madrid: Gredos, 2007.

GONZÁLES, Angel - "La fascinación del argumento ontológico". Revista de filosofía, Vol. VI, n. 9 (1993), pp. 201-206.

JOVEN ÁLVAREZ, Fernando - “Creer y entender en la carta 120 de San Agustín”. Scripta Fulgentina: revista de teología y humanidades, vol. XXII, no 43-44 (2012), pp. 197-211.

JOLIVET, Jean - La filosofía medieval en occidente. México: Siglo XXI editores, 1985.

MARÍAS, Julián - San Anselmo y el insensato: y otros estudios de filosofía. Madrid: Revista de Occidente, 1994.

ÓSCAR DÍEZ, Ricardo - “¿Si hay Dios, quién es?: una cuestión planteada por San Anselmo de Cantorbery en el Proslogion". Cuadernos de anuario filosófico. Serie Universitaria 136 (2001), pp. 101-150. 
ÓSCAR DÍEZ, Ricardo - Anselmo de Aosta ayer, hoy y mañana. Buenos Aires: Academia Nacional de Ciencias de Buenos Aires, 2009.

ÓSCAR DÍEZ, Ricardo - “Características del pensamiento Medieval”. In Vita Flumen - 10 años en el pensamiento medieval. Buenos Aires: Academia Nacional de Ciencias de Buenos Aires, 2015.

PLANTINGA, Alvin - The ontological argument: from St. Anselm to Contemporary Philosophers. New York: A Doubleday Anchor Original, 1965.

PÉREZ DE LABORDA, Miguel - Anselmo de Canterbury Esencial. Mataró: Montesinos-Esencial, 2010.

REINA-VALERA - Biblegateway [En línea]. 1960. [Accedido en 23 March 2021]. Disponible en https://www.biblegateway.com/passage/?search=\%C3\%89xodo\%203\%3A14\&version=RVR1960.

VELARDE, Julián - “Introducción”. In SAN ANSELMO - Obras Completas. Vol. I. Madrid: BAC, 2008, pp. 35-68.

VIGNAUX, Paul - El pensamiento en la Edad Media. México: Fondo de cultura Económica, 1983.

ZAÑARTU, Sergio - “Algunas notas sobre la búsqueda racional de Dios por Anselmo”. Anales de la Sociedad Chilena de Teología 3 (2003), pp. 125-143.

\section{NOTAS}

1. Estas nominaciones están íntimamente ligadas con una perspectiva ontológica del lenguaje. Existen varios estudios que interpretan la filosofía anselmiana desde esta disciplina. Cfr. SAN ANSELMO -Fragmentos sobre filosofía del lenguaje. Bogotá: Ediciones Uniandes - CESO, 2001. Asimismo, SAN ANSELMO - "Fragmentos sobre filosofía del lenguaje. Sobre el poder y la impotencia, la posibilidad y la imposibilidad, la necesidad y la libertad." In ANSELMO DE CANTERBURY - Fragmentos sobre filosofía del lenguaje. Bogotá: Ediciones Uniandes - CESO, 2001, pp. 159-211.

2. ÓSCAR DÍEZ, Ricardo - “¿Si hay Dios, quién es?: una cuestión planteada por San Anselmo de Cantorbery en el Proslogion". Cuadernos de anuario filosófico. Serie Universitaria 136 (2001), pp. 101-150.

3. San Anselmo en el proemio al Proslogion refiere que la sentencia Fides quaerens intellectum en realidad era el título original a este argumento, no obstante, posteriormente cambió al título que ahora conocemos, ya que anecdóticamente el abad de Bec quería mantener el anonimato de dicho manuscrito. Otra cuestión importante digna de mención se encuentra en el estudio realizado por Miguel Pérez de Laborda titulado Anselmo de Canterbury - Esencial donde el autor detalla como para él San Anselmo sientas las bases del método escolástico que se gestaría años después. Este dato es sumamente revelador en cuanto a la metodología o dialéctica anselmiana, puesto que marca la relación de armonía o tensión entre la fe/razón.

4. La identidad aquí aludida siempre quedará referida a Dios. Como se desarrollará a lo largo del artículo se demostrará como se edifica esta identidad entre verdad y ser.

5. Este manuscrito es posterior al argumento del Proslogion y en éste a diferencia del segundo San Anselmo desarrolla todo un ejercicio meditativo sobre la definición de verdad y los tipos de verdad que están orientados o debajo de la suma verdad. Cfr. SAN ANSELMO - "De Veritate". In SAN ANSELMO - Obras Completas. Vol. I. Madrid: BAC, 2008, pp. 487-535.

6. Este ejercicio intelectivo no es otra cosa que el esclarecimiento racional de la fe, sintetizado en el fides quaerens intellectum.

7. Un antecedente a esta identidad entre ser y verdad se haya internamente en el significado de la palabra y el valor de verdad que puede tomar esta. En sentido estricto, San Anselmo se ocupa 
ampliamente de este problema en el capítulo II del De Veritate. Cfr. SAN ANSELMO - "De Veritate". In SAN ANSELMO - Obras completas. Vol. I. Madrid: BAC, 2008, pp. 487-535.

8. Recordemos que la mayoría de los pensadores medievales y específicamente San Anselmo no dudaban de la verdad acerca de la existencia de las cosas, puesto que Dios no puede crear cosas falsas.

9. SAN ANSELMO - "Proslogion". In SAN ANSELMO - Obras completas. Vol. I. Madrid: BAC, 2008, p. 368.

10. GILSON, Étienne - La filosofía en la Edad Media. Madrid: Gredos, 2007.

11. E. Gilson abrió toda una nueva perspectiva en cuanto a los estudios medievales se refieren. De esta manera, los estudiosos contemporáneos de este periodo histórico han atribuido las categorías del realismo a la filosofía anselmiana. Lo interesante a esta denominación filosófica, es que en justa medida los medievales nunca utilizaron dichos términos, esto es, San Anselmo nunca considero su pensamiento en tanto realista, ni a su vez su contraparte nominalista como lo fue Roscelino. Cfr. GILSON, Étienne - El realismo metódico. Madrid: Rialp, 1974.

12. SAN ANSELMO - "Proslogion". In SAN ANSELMO - Obras completas, p. 366.

13. SAN ANSELMO - "Proslogion". In SAN ANSELMO - Obras completas, p. 366.

14. Por este término entendemos un solo ser que posee determinadas características que si bien otros objetos o cosas (res) pueden participar de ellas, el ser unívoco es el único que las posee de modo absoluto, sin responder a contingencias múltiples y/o accidentales.

15. He aquí la identidad expresa entre ser y verdad buscada por la propuesta aquí sugerida.

16. SAN ANSELMO - "De Veritate". In SAN ANSELMO - Obras completas.

17. SAN ANSELMO - "De Veritate". In SAN ANSELMO - Obras completas, p. 492.

18. Otro texto anselmiano a considerar es el De grammatico, anterior aún a De Veritate, pero que puede servir para terminar de corregir los excesos del lenguaje que se tornan contradictorios.

19. ÓSCAR DÍEZ, Ricardo - “¿Si hay Dios, quién es?”, p. 37.

20. Las sagradas escrituras son el pilar y la fuente del pensamiento medieval. Así, no es extraño que el abad de Bec al inicio del Proslogion no dude en citar esta fuente textual, más específicamente los salmos. No obstante, es interesante señalar la disparidad suscitada en algunos textos especializados al respecto de la metodología y filosofía anselmiana comparados con el discurso del propio San Anselmo. Primeramente Miguel Pérez de Laborda asiente en demostrar la insuficiencia de la exclusiva racionalidad en el modo de explicar lo real: "Quien llega racionalmente a afirmaciones opuestas a la fe es porque su modo de argumentar en realidad no es suficientemente racional (...) La fe, por tanto, ayuda a la razón señalándole cuándo ha errado el camino". PÉREZ DE LABORDA, Miguel - Anselmo de Canterbury - Esencial: existe algo mayor que lo cual nada pueda pensarse. Mataró: Montesinos / esencial, 2010, p. 25. Otro estudio especializado en Anselmo permite hacer una comparación interesante en esta modalidad metodológica, sin embargo, a nuestra interpretación parece no tener la misma orientación que el primero: “(...) incluso las palabras mismas de San Anselmo esclarecerían su fin: manifestar cómo es posible una prueba necessitas rationis sin la autoridad de la Sagrada Escritura. La definición de Dios, no es ni mucho menos bíblica, no deriva de la revelación; ya que en el Monologion aparece como algo natural e independiente de cualquier intervención de la fe, la concepción de un Dios trinitario cuya analogía con el proceso trinitario no eleva el discurso al plano de la fe.". GARCÍA DE LOMAS MIER, José María - "El Intellectus Fidei en San Anselmo de Canterbury: claves hermenéuticas de las relaciones fe-razón en el método intelectual anselmiano". Cuadernos de Filosofía, Excerpta e dissertationibus in philosophia 4 (1994), pp. 97-185. Pero ahora bien, si se realiza una comparación con las palabras del Santo Doctor, es en el prólogo al Monologion donde se 
percibe esa intención de no apoyarse en la autoridad de las Sagradas Escrituras, pero sin dejar de objetar una respuesta a los simples y necios que niegan a Dios. Cfr. SAN ANSELMO - “Monologion”. In SAN ANSELMO - Obras completas. Vol. I. Madrid: BAC, 2008, pp. 185-347.

21. Aquí Anselmo se dirige al insipiens. Este insensato es aquel que niega en su corazón a Dios. Antes de redactar el capítulo IV, San Anselmo anticipaba una respuesta en el capítulo III contra esta figura, citando directamente el Salmo $(14,1)$ “Por qué, entonces, «dijo el insensato en su corazón: no hay Dios» (...) siendo así que están evidente para la mente racional que tú eres, entre todos, de la manera más grande? ¿Por qué, sino porque es necio e insensato?”. SAN ANSELMO Proslogion. Con las réplicas de Gaunilón y Anselmo. Madrid: Tecnos, 2009, p. 80. Conviene advertir dos posturas semejantes a esta negación o imposibilidad de Dios. La primera puede resumirse en la figura del ateo, es decir, quién niega inmediatamente la existencia de Dios. No obstante, la réplica que esgrime San Anselmo no es de este orden. La objeción señalada por Anselmo está dirigida al hermano creyente que no acepta esta vía nominativa de aquello mayor que lo cual nada pueda pensarse. Como podrá notarse el insensato es un monje benedictino que responde al nombre de Gaunilón, por ende, acepta su condición de fe. A consecuencia de ello, la discusión se torna en una ruptura interna a la propia comunidad monástica, es decir, la discusión se encuentra en el orden entre creyentes.

22. Siguiendo el orden expositivo, en el apartado tocante a la cuarta y quinta nominación se explicará esta definición de verdad.

23. Ricardo Óscar Díez señala en la introducción a “¿Si hay Dios” la recepción cartesiana y kantiana del argumento anselmiano, evidenciando las limitaciones así de los condicionamientos históricos propios a la época moderna. Dicha parcialidad se puede encontrar más o menos presente en los mismos manuales de historia de las doctrinas filosóficas. Al respecto conviene revisar los textos históricos del medievalista francés Étienne Gilson. Sin embargo, una lectura más detallada revela las contradicciones de aquellas lecturas generales. Por ejemplo, pueden tenerse en cuenta los siguientes estudios. Este estudio toma más una aproximación al problema: MARÍAS, Julián - San Anselmo y el insensato y otros estudios de filosofía. Madrid: Revista de Occidente, 1944. Este segundo es más fiel en su lectura a las intenciones del autor medieval: PLANTINGA, Alvin - The ontological argument: from St. Anselm to Contemporary Philosophers. New York: A Doubleday Anchor Original, 1965.

24. Dicho pasaje se encuentra en el capítulo III. El ideal de la razón pura. Sección cuarta. Imposibilidad de una prueba ontológica de la existencia de Dios.

25. Cfr. VELARDE, Julián - "Introducción". In SAN ANSELMO - Proslogion. Con las réplicas de Gaunilón y Anselmo. Madrid: Tecnos, 2009.

26. VELARDE, Julián - “Introducción”, p. 43.

27. VELARDE, Julián - "Introducción”, p. 45.

28. Dicho gozo queda satisfecho después del pedido que clama el creyente a Dios. Especialmente las nominaciones reveladas, es decir, la cuarta y quinta nominación dan cuenta de esta respuesta. Cfr. ÓSCAR DÍEZ, Ricardo - “Si hay Dios, quién es?.

29. Esta fue la posterior interpretación cartesiana del argumento. Habrá que recordar que para René Descartes a través de la duda metódica quiere demostrar bajo ideas claras y distintas una realidad apoyada en una sola verdad, concluyendo que Dios es el ser perfecto. La tercera y quinta meditación demuestran la existencia de Dios, al igual que en Discurso del Método dedica una reflexión al problema de Dios. Cfr. DESCARTES, René - "Meditaciones metafísicas". In DESCARTES, René - Meditaciones metafísicas, Conversación con Burman, Correspondencia con Isabel de Bohemia. Barcelona: Gredos, 2014.

30. A lo largo del texto del Proslogion, San Anselmo utiliza el término latino in re, con el cual alude a la realidad de las cosas. Esta realidad se refiere a la existencia de los objetos, en otras palabras, 
es la realidad efectiva, no la "realidad" conceptual. Asintiendo en estos dos tipos de realidad, la palabra cae en la categoría del pensar mientras que las cosas (res) en la categoría del existir.

31. ÓSCAR DÍEZ, Ricardo - “Si hay Dios, quién es?, p. 76.

32. SAN ANSELMO - "Proslogion". In SAN ANSELMO - Obras completas, p. 358.

33. SAN ANSELMO - "Proslogion". In SAN ANSELMO - Obras completas, p. 368.

34. SAN ANSELMO - "Proslogion". In SAN ANSELMO - Obras completas, p. 370.

35. SAN ANSELMO - "Proslogion". In SAN ANSELMO - Obras completas, p. 370.

36. SAN ANSELMO - "Proslogion". In SAN ANSELMO - Obras completas, p. 369.

37. SAN ANSELMO - "Proslogion". In SAN ANSELMO - Obras completas, p. 369.

38. Más adelante en la tercera nominación los atributos divinos se orientan hacia una negación de todo aquello que el pensamiento ha podido inteligir, por ello, en esta segunda nominación todavía puede hablarse de un movimiento positivo previo al negativo. Sin embargo, conviene precisar que el valor de verdad de cada atributo seguirá siendo verdadero, ya que la limitación del inteligir queda reducida a la capacidad del pensamiento no al modo en el que se juzgan los atributos de Dios. Consecuentemente, los atributos divinos siempre son verdaderos.

39. Cfr. ÓSCAR DÍEZ, Ricardo - “¿Si hay Dios, quién es?, p. 39.

40. Cfr. ÓSCAR DÍEZ, Ricardo - “¿Si hay Dios, quién es?, p. 74.

41. El término de unificar no debe entenderse en una "unión" efectiva del pensamiento con la cosa (res), en otras palabras, el pensar solo mira intelectivamente las cosas, juzgando su valor de verdad o falsedad si la significación de la palabra coincide o no con lo percibido.

42. Estos momentos guardan cierta analogía con las intenciones vertidas en el proemio al Proslogion. Una lectura cuidadosa de este apartado demuestra casi a la mitad del primer párrafo la intención por parte de Anselmo en demostrar con un solo argumento que demostrase la verdad de Dios, que es el sumo bien y además lo que se cree de la substancia divina. Entonces, si se contrastan éstos con los aquí resumidos podrá notarse que hay una gran cercanía entre las dos primeras nominaciones con la verdad del ser de Dios, lo cual, asume la identidad entre ser-verdad aquí sugerida.

43. SAN ANSELMO - "Proslogion". In SAN ANSELMO - Obras completas, p. 358.

44. Esta nominación la enuncia San Anselmo en el primer párrafo al cap. II del Proslogion, inscribiendo una relación argumentativa de lo que ya había explicado en su Proemio sobre la Verdad de Dios, y ahora lo remite a la imposibilidad del pensamiento sobre un ser mayor a éste mismo, Ser que como ningun otro está por encima de todos: "Ergo Domine, qui das fidei intellectum, da mihi, ut quatum seis expedire intelligam, quia es sicut credimus, et hoc es quod credimus. Et quidem credimus Te esse aliquid quo nihil maius cogitari possiti" - SAN ANSELMO - "Proslogion". In SAN ANSELMO - Obras completas, p. 366.

45. Esta segunda nominación del cap. $\mathrm{V}$ al igual que la primera del cap. II, es un momento explicativo del objetivo trazado por Anselmo en su Proemio. Recordemos que en el primer párrafo de este apartado anterior al cap. I Anselmo insiste en pensar si es posible encontrar una sola prueba que no necesite ninguna otra y que demuestre al menos tres cosas, que Dios es verdadero, que es el sumo bien y que es aquello que creemos de la substancia divina. “(...) quia Deus vere est, et quia est summum bonum nullo alio indigens, et quo omnia indiget ut sint, et ut bene sint et quaecumque de divina credimus substantia, sufficeret". SAN ANSELMO - "Proslogion". In SAN ANSELMO - Obras completas, pp. 358-370.

46. ÓSCAR DÍEZ, Ricardo - “¿Si hay Dios, quién es?, p. 82.

47. SAN ANSELMO - "Proslogion". In SAN ANSELMO - Obras completas, p. 386.

48. La incapacidad intelectiva viene dada en el reconocimiento del paso de lo inteligible a lo ininteligible. Intentar demostrar todo lo real racionalmente es el modo del pensamiento moderno. La filosofía anselmiana vista desde la sentencia fides quaerens intellectum va mostrando esos mecanismos del pensar que se hayan sostenidos por la base de la creencia. La fe es el sostén a todo el edificio edificado del pensamiento que se va construyendo en su ascenso a Dios. Sin 
embargo, es importante advertir como el mismo argumento del Proslogion va haciendo ese mismo asciendo anteponiendo los límites del pensar con las nominaciones reveladas.

49. La frase anselmiana por excelencia tiene ecos del pensamiento de San Agustín de Hipona.

50. La frase agustina "creer para entender, y entender para creer" hace un eco en el argumento anselmiano del Proslogion en el capítulo I. San Anselmo toma la primera parte de esta sentencia e inaugura todo el proceso intelectivo del fides quaerens intellectum bajo la frase "quia nisi credidero, non intelligam". SAN ANSELMO - "Proslogion". In SAN ANSELMO - Obras completas, p. 366). Conviene revisar la originalidad de estas frases en cada uno de estos pensadores aquí citados. Sobre San Agustín Cfr. JOVEN ÁLVAREZ, Fernando - "Creer y entender en la carta 120 de San Agustín". Scripta Fulgentina: revista de teología y humanidades XXII/43-44 (2012), pp. 197-211.

51. Esta experiencia religiosa la advierte Ricardo O. Díez en ¿Si hay Dios quién es? Respecto a este estudio aplicado al argumento del Proslogion el autor citado hace nota a dos textos cuyas referencias permitirán ahondar con mayor precisión a este modo del acceso divino. El primero intitulado Lo santo de Rudolph Otto. El segundo De visiones Dei de Nicolás de Cusa.

52. Mutua identidad entre el ser de Dios y la verdad de Dios. Esta es la identidad propuesta por esta exposición entre el Proslogion-De Veritate.

53. En el volumen I a la edición de los textos anselmianos de la editorial Biblioteca de Autores Cristianos se encuentra un apartado titulado "la suma teológica anselmiana". Es interesante notar el anacronismo a este título, pues como se sabe San Anselmo nunca elaboró dicho manuscrito. Sin embargo, se puede interpretar que tal texto atiende una serie de referencias a las obras del Santo Doctor.

54. Su discípulo Eadmero narra esta situación anecdótica en la biografía que hizo de su Maestro. Cfr. EADMERO - "Vida de San Anselmo". In SAN ANSELMO - Obras completas. Vol. I. Madrid: BAC, 2008, pp. 5-73.

55. SAN ANSELMO - "Proslogion". In SAN ANSELMO - Obras completas, p. 394.

56. Existe un cambio sustancial tanto en las motivaciones como metodología que llevaron a San Anselmo a redactar el argumento del Proslogion respecto a su obra antecesora del Monologion. A diferencia del segundo, en el primero puede y no duda en citar la Sagrada Escritura, puesto que puede advertirse la gran cantidad de citas bíblicas que utiliza en el capítulo I. Específicamente en esta cuarta nominación Anselmo tiene presente el Antiguo Testamento.

57. REINA-VALERA - Biblegateway [En línea]. 1960. [Accedido en 23 March 2021]. Disponible en https://www.biblegateway.com/passage/?search=\%C3\%89xodo\%203\%3A14\&version=RVR1960

58. Cfr. ÓSCAR DÍEZ, Ricardo - “¿Si hay Dios, quién es?”. R. Díez explica con mayor detalle este cambio pronominal que realizó San Anselmo en esta cuarta nominación.

59. SAN ANSELMO - "Proslogion". In SAN ANSELMO - Obras completas, p. 396.

60. El argumento del Proslogion termina por nominar con el Nuevo Testamento, suponiendo una ascensión nominativa del intelecto a la revelación. La trinidad fue un concepto que marca sustancialmente la filosofía anselmiana. Una primera aproximación a las tres personas de una sola naturaleza se encontraba ya en el Monologion. Posteriormente, en el Proslogion cap. XXIII. Sin embargo, en otros textos del Santo Padre se continua la reflexión filosófica acerca de su naturaleza, pues existió la disputa directa contra el nominalismo de Roscelino de Compiègne. Conviene tener presente la epístola sobre la encarnación del verbo, texto donde se rebatió tal discusión.

61. ÓSCAR DÍEZ, Ricardo - “Si hay Dios, quién es?”, p. 146. 


\section{RESÚMENES}

San Anselmo de Canterbury fue un monje benedictino que redactó el argumento del Proslogion en el siglo XI. Las nominaciones o nombres de Dios, son los diferentes modos por los cuales San Anselmo explicó los atributos divinos. A partir de una reinterpretación al estudio de Ricardo 0. Díez en ¿Si hay Dios, quién es? es propuesta una identidad entre el ser y la verdad de Dios, implicando así una mutua relación entre estos atributos con la definición de verdad anselmiana. La sentencia fides quaerens intellectum representará una metodología o modelo a través de la cual se orienta el pensamiento en el esclarecimiento racional de estas nominaciones divinas. No obstante, la misma búsqueda intelectiva reconocerá una limitación racional frente aquello que cree por fe, sentencia heredada por San Agustín en la filosofía del Santo Doctor Anselmo. Desde nuestra reflexión remarcaremos como las nominaciones del Proslogion y la definición de la verdad del De Veritate permiten pensar en una identidad que relaciona ambas obras del pensamiento anselmiano.

Saint Anselm of Canterbury was a Benedictine monk who wrote the Proslogion argument in the 11th century. The nominations or names of God, are the different ways by which Saint Anselm explained the divine attributes. From a reinterpretation of Ricardo O. Díez's study ¿Si hay Dios, quién es? an identity between the being and the truth of God is proposed, thus implying a mutual relationship between these attributes with the Anselmian definition of truth. The sentence fides quaerens intellectum will represent a methodology or model through which the thought is oriented in the rational clarification of these divine nominations. However, the same intellectual search will recognize a rational limitation against what it believes by faith. The nominations of the Proslogion and the definition of the truth of the De Veritate allow us to think of an identity that relates both works of Anselmian thought, identity that this article wanted to propose.

\section{ÍNDICE}

Keywords: Saint Anselm, Proslogion, De Veritate, identity, fides quaerens intellectum

Palabras claves: San Anselmo, Proslogion, De Veritate, identidad, fides quaerens intellectum

\section{AUTOR}

\section{JOSÉ LUIS GAONA CARRILLO}

Universidad Michoacana de San Nicolás de Hidalgo. Instituto de Investigaciones Filosóficas Luis Villoro, 58260 Morelia, México. jose.gaona@umich.mx 